https://doi.org/10.15407/ujpe63.9.828

R.M. BALABAI, A.V. ZDESHCHYTS

Kryvyi Rih State Pedagogical University

(54, Gagarin Ave., Kryvyi Rih 50085, Ukraine; e-mail: balabai@i.ua, bodehita@gmail.com)

\title{
NANOCELLULOSE AS THE MAIN COMPOSITE COMPONENT OF ELECTROMECHANICAL SENSORS ${ }^{1}$
}

\begin{abstract}
Within the methods of the electron density functional and the ab initio pseudopotential, we have obtained the spatial distributions of the density of valence electrons, density of electron states, band gap, valence band, and charge for the cellulose-based model composite structures under mechanical influences, using authors' program complex. It is determined that the electronic properties of composite structures based on nanocellulose can be controlled, for example, by changing the distance between the layers of composite components that happens during a mechanical compression or stretching.
\end{abstract}

Ke y words: heterocomposites, nanocellulose, graphene, electron density functional, ab initio pseudopotential.

\section{Introduction}

Modern electronic devices are usually made from materials which cannot be biodegradable and sometimes potentially toxic. At present, their production includes processes that require high vacuum and high temperature, i.e., costly operations [1].

However, the service life of electronics is becoming increasingly shorter. This creates not only technological, but also increasing environmental problems [2].

In this regard, the promising direction is the use of cellulose as a substrate and a functional element of electronics. Half the biomass produced by photosynthetic organisms such as plants, algae, and some bacteria consists of cellulose, which is the most common molecule on the planet. This is a renewable, portable, and flexible material [3].

Deformation sensors are used to detect an electrical displacement under mechanical deformations and are widely used in the automotive industry, mechanical engineering, in sensors of various types, etc. In works $[4,5]$, the mechanical properties of a piezoresistive strain gauge based on a graphene/nanocellulous nanopaper and cellulose/graphene sensory material are studied. However, theoretical knowledge of the electronic properties of crystalline nanocellulose (CNC) composite structures does not exist completely. For the expansion of information about the electronic properties of composite structures with nanocellulose, such as the density of electronic states,

(C) R.M. BALABAI, A.V. ZDESHCHYTS, 2018 the bandgap, and the core charge under a mechanical compression, we will calculate them by means of the electron density functional methods and the firstprinciples pseudopotential, by using our own program code [6].

\section{Methods of Research}

The ground states of the electron-nucleus systems are obtained, by finding a self-consistent-field solution of the Kohn-Sham equations. Electronic variables are only determined with the fixed atomic cores. Following the Kohn-Sham procedure, the electronic density can be written in terms of occupied orthonormal one-particle wave functions:

$n(\mathbf{r})=\sum_{i}\left|\psi_{i}(\mathbf{r})\right|^{2}$

The point on the surface of potential energy in the Born-Oppenheimer approximation is determined as a minimum energy functional with regard to the wave functions:

$E\left[\left\{\psi_{i}\right\},\left\{R_{j}\right\},\left\{\alpha_{\nu}\right\}\right]=\sum_{i} \int_{\Omega} d^{3} r \psi_{i}^{*}(\mathbf{r}) \times$ $\times\left[-\frac{\hbar^{2}}{2 m} \nabla^{2}\right] \psi_{i}(\mathbf{r})+U\left[\{n(\mathbf{r})\},\left\{R_{j}\right\},\left\{\alpha_{\nu}\right\}\right]$,

where $\left\{R_{j}\right\}$ are coordinates of atomic cores; $\left\{\alpha_{\nu}\right\}$ are any external influences on the system.

${ }^{1}$ The paper was presented at the XXIII Galyna Puchkovska International School-Seminar "Spectroscopy of Molecules and Crystals".

ISSN 2071-0194. Ukr. J. Phys. 2018. Vol. 63, No. 9 
In the generally accepted formulation, the minimization of the energy functional (2) with respect to one-particle orbitals with additional orthonormal constraint on the one-particle orbitals $\psi_{i}(\mathbf{r})$ results in Kohn-Sham one-particle equations:

$\left\{-\frac{\hbar^{2}}{2 m} \nabla^{2}+\frac{\partial U}{\partial n(\mathbf{r})}\right\} \psi_{i}(\mathbf{r})=\varepsilon_{i} \psi_{i}(\mathbf{r})$.

In the solution of these equations, the pseudopotential formalism was used, according to which a solid is considered as a set of valence electrons and the ion cores. In the pseudopotential approximation, the operator of the pseudopotential $V_{P S}$, which describes the interaction of valence electrons with the core, is small, and the corresponding pseudowavefunction is smooth. Pseudopotentials are required to correctly represent the long-range interactions of the core and to produce pseudowavefunction solutions that approach the full wavefunction outside a core radius $r_{c}$. In addition, it is necessary that a pseudopotential be transferable. This means that the same pseudopotential can be used in calculations of different chemical environments resulting in calculations with comparable accuracy. For example, Bachelet, Hamann, and Schliiter proposed an analytic fit to the pseudopotentials. This $a b$ initio pseudopotential will be used in the present work.

The full crystalline potential is constructed as a sum of ion pseudopotentials that do not overlap and are associated with ions (nucleus + core electrons) located at the $\mathbf{R}_{S}$ positions which are periodically repeated for crystals:

$V_{\text {cryst }}(\mathbf{r}) \rightarrow V_{P S}(\mathbf{r})=\sum_{\mathbf{p}} \sum_{S} \hat{V}_{S}^{P S}\left(\mathbf{r}-\mathbf{p}-\mathbf{R}_{S}\right)$.

For nonperiodic systems, such as a thin film or a cluster, the problem of the lack of periodicity is circumvented by the use of the supercell method. Namely, the cluster is periodically repeated, but the distance between each cluster and its periodic images is so large that their interaction is negligible. The ubiquitous periodicity of a crystal (or artificial) lattice produces a periodic potential and thus imposes the same periodicity on the density (implying Bloch's theorem). The Kohn-Sham potential of a periodic system exhibits the same periodicity as a direct lattice, and the Kohn-Sham orbitals can be written in the Bloch form:

$\psi(\mathbf{r})=\psi_{i}(\mathbf{r}, \mathbf{k})=\exp (i \mathbf{k} \mathbf{r}) U_{i}(\mathbf{r}, \mathbf{k})$ where $\mathbf{k}$ is a vector in the first Brillouin zone. The functions $u_{i}(\mathbf{r}, \mathbf{k})$ have the periodicity of a direct lattice. The index $i$ runs over all states. The periodic functions $u_{i}(\mathbf{r}, \mathbf{k})$ are expanded in the plane wave basis. This heavily suggests to use plane waves as the generic basis set in order to expand the periodic part of the orbitals. Since the plane waves form a complete orthonormal set of functions, they can be used to expand orbitals according to:

$\Psi_{J}(\mathbf{k}, \mathbf{r})=\frac{1}{\sqrt{N_{0}} \sqrt{\Omega}} \sum_{\mathbf{G}} b_{J}(\mathbf{k}+\mathbf{G}) \exp (i(\mathbf{k}+\mathbf{G}) \mathbf{r})$,

where $\mathbf{G}$ is a vector in the reciprocal space, $\Omega$ is the volume of elemental cells, which consists of a periodic crystal or an artificial superlattice, when reproducing nonperiodic systems.

After the Fourier transform to the reciprocal space, Eq. (3) has the form:

$\sum_{\mathbf{G}}\left[\left\{\frac{\hbar^{2}}{2 m}(\mathbf{k}+\mathbf{G})^{2}-\varepsilon_{j}\right\} \delta_{\mathbf{G}, \mathbf{G}^{\prime}}+\right.$

$\left.+V_{\mathrm{KS}}\left(\mathbf{k}+\mathbf{G}, \mathbf{k}+\mathbf{G}^{\prime}\right)\right] b_{j}(\mathbf{k}+\mathbf{G})=0$,

where $V_{\mathrm{KS}}$ is the Kohn-Sham potential:

$V_{\mathrm{KS}}\left(\mathbf{k}+\mathbf{G}, \mathbf{k}+\mathbf{G}^{\prime}\right)=V_{p s}\left(\mathbf{k}+\mathbf{G}, \mathbf{k}+\mathbf{G}^{\prime}\right)+$

$+V_{H}\left(\mathbf{G}^{\prime}-\mathbf{G}\right)+V_{X C}\left(\mathbf{G}^{\prime}-\mathbf{G}\right)$,

and $V_{X C}$ is the exchange-correlation potential. To calculate it, we used Ceperley-Alder's LDA approximation that has been parametrized by Perdew and Zunger. It is well known that a choice of the exchange-correlation functional predetermines a band gap $[7,8]$. Nevertheless, LDA in DFT does reproduce a lot of the basic physics, but, without some fine tuning, it generally provides no details of the experimental band structure correctly. By carrying out a comparative analysis of the behavior of the electron-structural characteristics of nanosystems during the transition from one configuration to another one, we give the opportunity to avoid the imperfection of LDA and to reveal reliable information.

In the general case, the expressions describing the potentials of interactions are complex. The use of the atomic bases containing the inversion operation in the point symmetry group leads to the fact that the Fourier-components in the expansion of all expressions are real. 
The main quantity in the formalism of the electron density functional is the charge density. It is estimated from a self-consistent solution of Eqs. (6) which should be performed at all points of the nonreduced section of the Brillouin zone:

$\rho(\mathbf{G})=\frac{2}{N_{T}} \sum_{\mathbf{k}} \sum_{\mathbf{j}} \sum_{\alpha \in T} \sum_{\mathbf{G}^{\prime}} b_{j}^{*}\left(\mathbf{k}+\mathbf{G}^{\prime}+\alpha \mathbf{G}\right) b_{j}\left(\mathbf{k}+\mathbf{G}^{\prime}\right)$,

where the index $j$ runs over all occupied states, $\mathbf{k}$ is a vector in the first Brillouin zone, $N_{T}$ is the number of the operators $\alpha$ in the point group $T$ of the atomic basis, and the factor 2 takes the spin degeneracy into account.

The estimated effort can be reduced, if there is the integral over the Brillouin zone to approximate by summing over special points of the Brillouin zone. It is possible to replace, with satisfactory precision, the summation over the finite number of special points to one point in the Brillouin zone. It is possible to distinguish only the $\Gamma$-point in the Brillouin zone, especially as it relates to the artificial periodic systems.

The distribution of electrons along the energy zones for the $\Gamma$-state of the investigated structures was found by means of a numerical calculation of the derivative $\lim _{\Delta E \rightarrow 0} \Delta N / \Delta E$ (where $\Delta N$ is a number of allowed states for the $\Delta E$ interval of energy). The one-particle energy spectrum was obtained from calculations of the eigenvalues of the Kohn-Sham matrix. In accordance with the ideology of electronic density functional, the occupied states at absolute zero temperature were defined. This allowed us to define the position of the last occupied state, number of occupied states, being half the number of electrons (due to ignoring the spin of the electron), and position of the first free states.

The result of calculations of the electronic structure of the polyatomic system within the theory of electronic density functional is the electron density, which is a continuous quantity normalized to the total number of electrons in the system (in our case, the total number of electrons in the unit cell of a super lattice). To estimate the redistribution of the electron charge between atoms, the charge in the surrounding region with volume $V_{\alpha}$ of an atom $\alpha$ was calculated:

$q_{\alpha}=Z_{\alpha}-\int_{V_{\alpha}} n(\mathbf{r}) d^{3}$.

\section{Atomic Systems and the Results of Their Calculation}

Since the calculation algorithm provides for translational symmetry in the explored atomic systems, the orthorhombic-type artificial superlattice was initially created. The researched systems are determined by parameters of the superlattice and the atomic basis. The dimensions of the unit cell in the $\mathrm{X}, \mathrm{Y}$, and $\mathrm{Z}$ directions were chosen to avoid the interaction of the translated cellulose/graphene composites $(\mathrm{CNC} / \mathrm{Gr})$. The calculations were made only for $\Gamma$ point of the Brillouin zone of an artificial supergrid. The response of the electronic subsystem of the composite on a mechanical impact was determined, by comparing the characteristics of such systems:

- system 1: the isolated nanocellulose fiber fragment - $\left(\mathrm{C}_{6} \mathrm{H}_{10} \mathrm{O}_{5}\right)_{3} \mathrm{H}_{3}$. To reproduce it, the atomic basis contained $18 \mathrm{C}$ atoms, $15 \mathrm{O}$ atoms, and 33 $\mathrm{H}$ atoms, 3 of which were terminated by the broken nanocellulose bonds; the symbol of this system is $(\mathrm{CNC})$;

- system 2: fragment of the graphene plane. To reproduce it, the atomic basis contained $96 \mathrm{C}$ atoms; the symbol of this system is $(\mathrm{Gr})$;

- system 3: the isolated fragment of a nanocelulose fiber with a three-atom copper cluster in its vicinity. To reproduce it, the atomic basis contained $18 \mathrm{C}$ atoms, $15 \mathrm{O}$ atoms, $33 \mathrm{H}$ atoms, and $3 \mathrm{Cu}$ atoms; the symbol of this system is $\left(\mathrm{CNC} / \mathrm{Cu}_{3}\right)$;

- system 4: fragment of the graphene plane, on which fragments of nanocellulose fibers are located on both sides of the composite. To reproduce it, the atomic basis contained $90 \mathrm{C}$ atoms, $30 \mathrm{O}$ atoms, and $66 \mathrm{H}$ atoms; the symbol of this system is $(2 \mathrm{CNC} / \mathrm{Gr})$. The distance between graphene and nanocellulose was equal to the length of the chemical bond between carbon atoms and a hydrogen equal to $1.1 \AA$ (such a composite was considered mechanically non-strained) (Fig. 1);

- systems 5, 6, and 7 were composites that originated from system 4, with a distance between the graphene plane and nanocelulose to be reduced by $3 \%, 4 \%$, and $5 \%$, respectively. The symbols of this system are $(2 \mathrm{CNC} / \mathrm{Gr}-3 \%),(2 \mathrm{CNC} / \mathrm{Gr}-4 \%)$, and $(2 \mathrm{CNC} / \mathrm{Gr}-5 \%)$ respectively; such composites were considered mechanically strained (compressed);

- system 8 was a composite that originated from system 4 , with an increase in the distance between the

ISSN 2071-0194. Ukr. J. Phys. 2018. Vol. 63, No. 9 
graphene plane and nanocelulose by $5 \%$. The symbol of this system is $(2 \mathrm{CNC} / \mathrm{Gr}+5 \%)$; such composite was considered mechanically strained (stretched);

- system 9: a fragment of a nanocelulose fiber on the graphene plane with a three-atom cluster of copper in its vicinity. To reproduce it, the atomic basis contained $90 \mathrm{C}$ atoms, $30 \mathrm{O}$ atoms, $66 \mathrm{H}$ atoms, and $6 \mathrm{Cu}$ atoms; the symbol of this system is $\left(2 \mathrm{CNC} / 2 \mathrm{Cu}_{3} / \mathrm{Gr}\right)$; the composite is mechanically non-strained;

- system 10: the composite that originated from system 9 , with an increase in the distance between the graphene plane and nanocelulose by $5 \%$. The symbol of this system is $\left(2 \mathrm{CNC} / 2 \mathrm{Cu}_{3} / \mathrm{Gr}+5 \%\right)$; such composite was considered mechanically strained (stretched).

Table 1 and Figs. 2 and 3 demonstrate the calculated values of the band gap $E_{g}$ and the valence band width $E_{v}$ of the nanocellulose-containing systems.

From the obtained results, it can be seen that the maximum width of the valence band belongs to the isolated fragment of a nanocelulose fiber with a threeatom copper cluster in its vicinity. Wherein, the number of electrons in the unit cell in this system is not the largest.

It is determined that the value of the band gap of an isolated nanocellulose fiber fragment is the largest in comparison with composites with any addition to nanocellulose of other materials such as graphene planes and copper atoms. The exception is the $(2 \mathrm{CNC} / \mathrm{Gr}+5 \%)$ system. The calculated value of the band gap of an isolated nanocellulose fiber fragment is in good agreement with the experimen-

Table 1. Calculated values

of the band gap $E_{g}$ and the valence band width $E_{v}$ of the nanocellulose-containing systems

\begin{tabular}{|c|l|c|c|}
\hline No. & \multicolumn{1}{|c|}{ System } & $\Delta E_{\nu}, \mathrm{eV} / \mathrm{e}^{-} \mathrm{e}^{-}$ & $E_{g}, \mathrm{eV}$ \\
\hline 1 & $\mathrm{CNC}$ & 17.01 & 4.63 \\
2 & $\mathrm{Gr}$ & 2.31 & 0.54 \\
3 & $\mathrm{CNC} / \mathrm{Cu}_{3}$ & 18.25 & 1.63 \\
4 & $2 \mathrm{CNC} / \mathrm{Gr}$ & 10.14 & 1.90 \\
5 & $2 \mathrm{CNC} / \mathrm{Gr}-3 \%$ & 10.13 & 1.09 \\
6 & $2 \mathrm{CNC} / \mathrm{Gr}-4 \%$ & 10.13 & 2.45 \\
7 & $2 \mathrm{CNC} / \mathrm{Gr}-5 \%$ & 10.12 & 2.72 \\
8 & $2 \mathrm{CNC} / \mathrm{Gr}+5 \%$ & 10.16 & 5.17 \\
9 & $2 \mathrm{CNC} / 2 \mathrm{Cu} / \mathrm{Gr}+5 \%$ & 9.14 & 0.68 \\
\hline
\end{tabular}

ISSN 2071-0194. Ukr. J. Phys. 2018. Vol. 63, No. 9

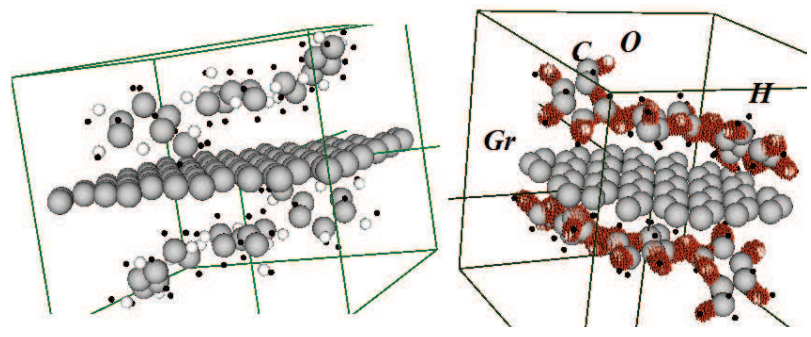

Fig. 1. Structure of a composite based on graphene and cellulose used for calculations (left); the spatial distribution of the valence electrons density within the interval of $1.0-0.9$ of the maximum value for $\mathrm{CNC} / \mathrm{Gr}$

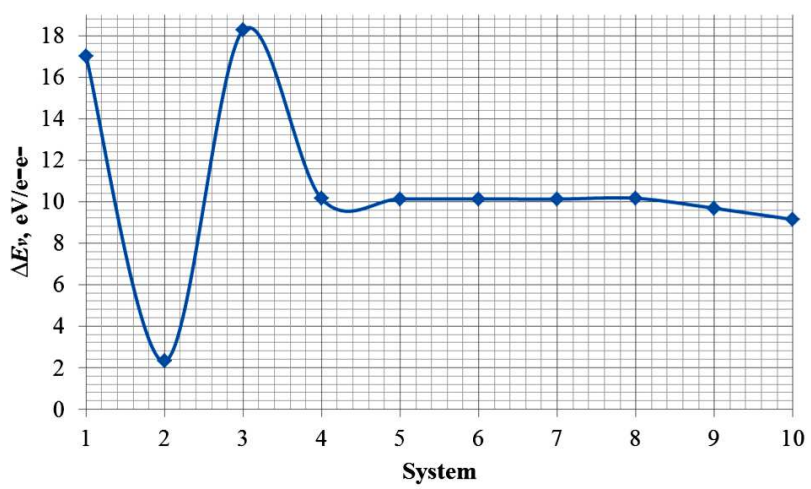

Fig. 2. Valence band width of systems 1-10, which are normalized to the number of electrons in a unit cell

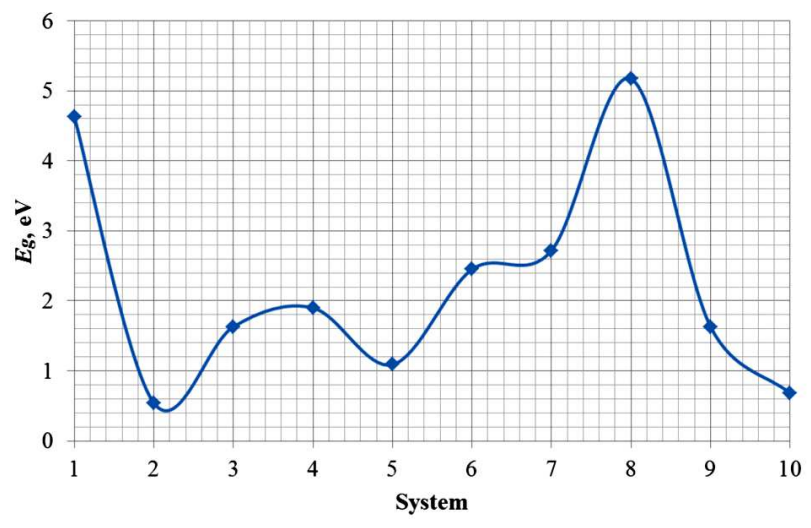

Fig. 3. Band gap width of systems 1-10

tal value $\left(E_{g} \approx 4.5 \mathrm{eV}\right)$, which is given in [9] for a nanofibrillated cellulose film.

The electronic properties of the composite structures based on nanocellulose can be controlled, for example, by changing the distance between the layers of the composite components, which happens dur- 

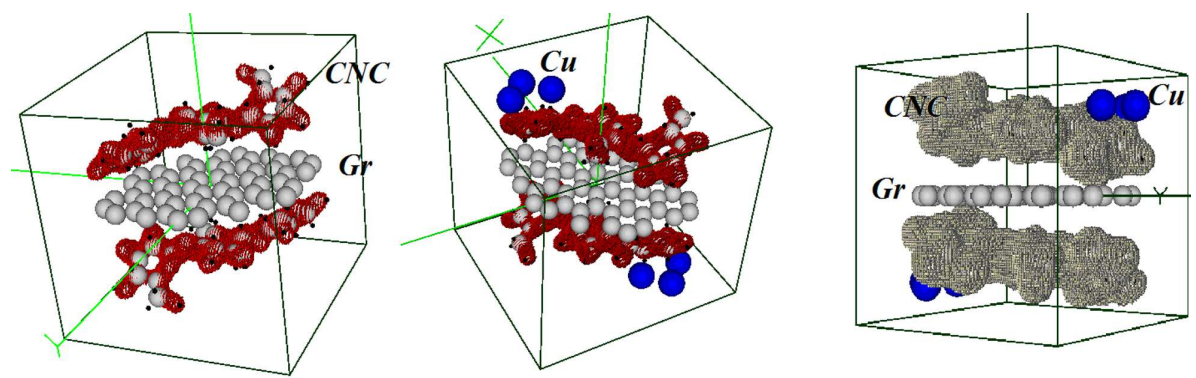

Fig. 4. The Spatial distributions of the density of valence electrons for $(2 \mathrm{CNC} / \mathrm{Gr})$ and $\left(2 \mathrm{CNC} / 2 \mathrm{Cu}_{3} / \mathrm{Gr}\right)$ systems within an interval of $0.8-0.7$ of the maximum value; and $\left(2 \mathrm{CNC} / 2 \mathrm{Cu}_{3} / \mathrm{Gr}\right)$ system within an interval of $0.2-0.1$ of the maximum value

Table 2. Values of the charge in a vicinity of the carbon core $(\mathrm{C})$ with radius of $0.73 \AA$

\begin{tabular}{|c|c|c|c|c|c|}
\hline Atom & CNC & $2 \mathrm{CNC} / \mathrm{Gr}$ & $2 \mathrm{CNC} / \mathrm{Gr}-3 \%$ & $2 \mathrm{CNC} / \mathrm{Gr}-4 \%$ & $2 \mathrm{CNC} / \mathrm{Gr}-5 \%$ \\
\hline 1 & 1.076 & 0.050 & 0.049 & 0.050 & 0.052 \\
2 & 1.536 & 0.511 & 0.568 & 0.585 & 0.599 \\
3 & 1.529 & 0.485 & 0.523 & 0.553 & 0.570 \\
4 & 1.477 & 0.692 & 0.720 & 0.725 & 0.732 \\
5 & 1.302 & 0.206 & 0.211 & 0.459 & 0.463 \\
6 & 1.406 & 0.407 & 0.460 & 0.035 & 0.031 \\
7 & 1.088 & 0.048 & 0.037 & 0.271 & 0.280 \\
8 & 1.441 & 0.257 & 0.264 & 0.395 & 0.405 \\
9 & 1.576 & 0.371 & 0.381 & 0.500 & 0.222 \\
10 & 1.425 & 0.477 & 0.491 & 0.221 & 0.229 \\
11 & 1.326 & 0.203 & 0.218 & 0.108 & 0.113 \\
12 & 1.372 & 0.235 & 0.231 & 0.218 & 0.218 \\
13 & 1.056 & 0.088 & 0.103 & 0.255 & 0.258 \\
14 & 1.473 & 0.206 & 0.215 & 0.151 & 0.154 \\
15 & 1.456 & 0.239 & 0.248 & 0.252 & 0.247 \\
16 & 1.398 & 0.144 & 0.147 & 0.407 & 0.406 \\
17 & 1.294 & 0.261 & 0.261 & 0.407 & \\
\hline
\end{tabular}

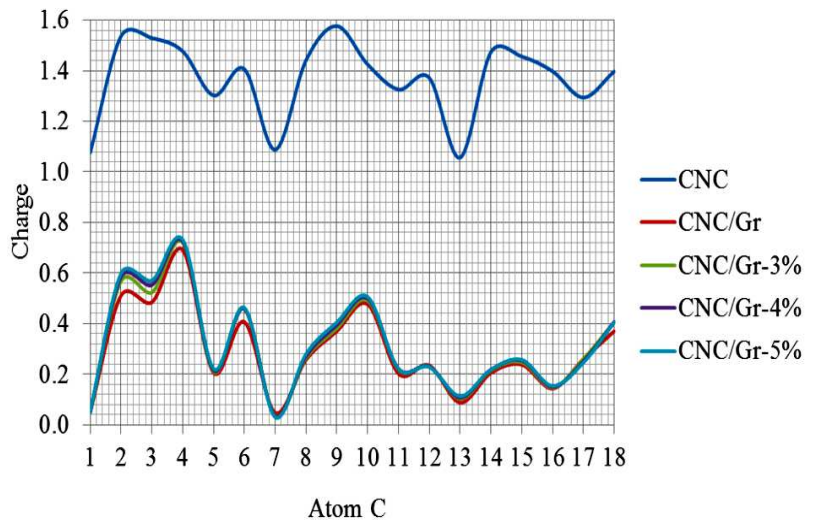

Fig. 5. Values of the charge in a vicinity of the carbon core for systems $1,4-7$ ing a mechanical compression or stretching. This is a physical mechanism for using nanocellulose/graphene composites as electromechanical sensors.

So, the valence band width decreases with the mechanical compression of the nanocellulose/graphene composite and grows with the stretching. The change in the band gap of such composites under a mechanical impact has the tendency to increase.

The spatial distributions of thedensity of valence electrons for the nanocellulose/graphene composite with the addition of copper are showed in Fig. 4.

The analysis of these spatial distributions of the density of valence electrons showed that the maximum density of the composite is concentrated around 
Table 3. Values of the charge in a vicinity of the hydrogen core $(H)$ with a radius of $0.37 \AA$

\begin{tabular}{|c|c|c|c|c|c|}
\hline Atom & $\mathrm{CNC}$ & $2 \mathrm{CNC} / \mathrm{Gr}$ & $2 \mathrm{CNC} / \mathrm{Gr}-3 \%$ & $2 \mathrm{CNC} / \mathrm{Gr}-4 \%$ & $2 \mathrm{CNC} / \mathrm{Gr}-5 \%$ \\
\hline 19 & 0.761 & 0.592 & 0.596 & 0.597 & 0.597 \\
\hline 20 & 0.789 & 0.663 & 0.676 & 0.677 & 0.680 \\
\hline 21 & 0.769 & 0.655 & 0.655 & 0.658 & 0.659 \\
\hline 22 & 0.672 & 0.570 & 0.566 & 0.566 & 0.565 \\
\hline 23 & 0.689 & 0.563 & 0.559 & 0.556 & 0.552 \\
\hline 24 & 0.778 & 0.690 & 0.694 & 0.694 & 0.695 \\
\hline 25 & 0.792 & 0.711 & 0.719 & 0.719 & 0.721 \\
\hline 26 & 0.758 & 0.630 & 0.627 & 0.626 & 0.625 \\
\hline 27 & 0.753 & 0.637 & 0.643 & 0.644 & 0.646 \\
\hline 28 & 0.691 & 0.595 & 0.598 & 0.599 & 0.600 \\
\hline 29 & 0.769 & 0.568 & 0.572 & 0.573 & 0.574 \\
\hline 30 & 0.762 & 0.612 & 0.616 & 0.618 & 0.619 \\
\hline 31 & 0.791 & 0.640 & 0.637 & 0.637 & 0.636 \\
\hline 32 & 0.687 & 0.569 & 0.568 & 0.568 & 0.567 \\
\hline 33 & 0.965 & 0.567 & 0.569 & 0.569 & 0.569 \\
\hline 34 & 0.776 & 0.655 & 0.657 & 0.658 & 0.660 \\
\hline 35 & 0.788 & 0.698 & 0.699 & 0.700 & 0.699 \\
\hline 36 & 0.761 & 0.631 & 0.626 & 0.627 & 0.628 \\
\hline 37 & 0.767 & 0.620 & 0.623 & 0.624 & 0.624 \\
\hline 38 & 0.716 & 0.616 & 0.617 & 0.618 & 0.620 \\
\hline 39 & 0.767 & 0.637 & 0.635 & 0.635 & 0.636 \\
\hline 40 & 0.758 & 0.591 & 0.588 & 0.588 & 0.586 \\
\hline 41 & 0.763 & 0.629 & 0.629 & 0.631 & 0.631 \\
\hline 42 & 0.692 & 0.585 & 0.586 & 0.584 & 0.583 \\
\hline 43 & 0.685 & 0.584 & 0.586 & 0.587 & 0.586 \\
\hline 44 & 0.790 & 0.527 & 0.516 & 0.514 & 0.511 \\
\hline 45 & 0.772 & 0.566 & 0.557 & 0554 & 0.550 \\
\hline 46 & 0.709 & 0.602 & 0.600 & 0.598 & 0.599 \\
\hline 47 & 0.753 & 0.620 & 0.615 & 0.612 & 0.611 \\
\hline 48 & 0.762 & 0.673 & 0.678 & 0.678 & 0.678 \\
\hline 49 & 0.700 & 0.590 & 0.579 & 0.576 & 0.575 \\
\hline
\end{tabular}

nanocellulose for all systems in various mechanical states of the composite.

Tables 2-4 and Figs. 5-7 show the calculated value of the charge in the surrounding region with volume $V_{\alpha}$ of atoms that belong to a nanocellulose fiber for systems $1,4-7$. Charges were estimated in a vicinity of the carbon core with a radius of $0.73 \AA$, hydrogen core with a radius of $0.37 \AA$, and oxygen one with a radius of $1.2 \AA$. The charge was in units of electron charge, the value of which was equal to 1 in the atomic system of units, which was used in the calculations.

As can be seen from Tables 2-4, the values of the charge in vicinities of the carbon cores, hydrogen and oxygen cores of nanocellulose decrease for all

ISSN 2071-0194. Ukr. J. Phys. 2018. Vol. 63, No. 9

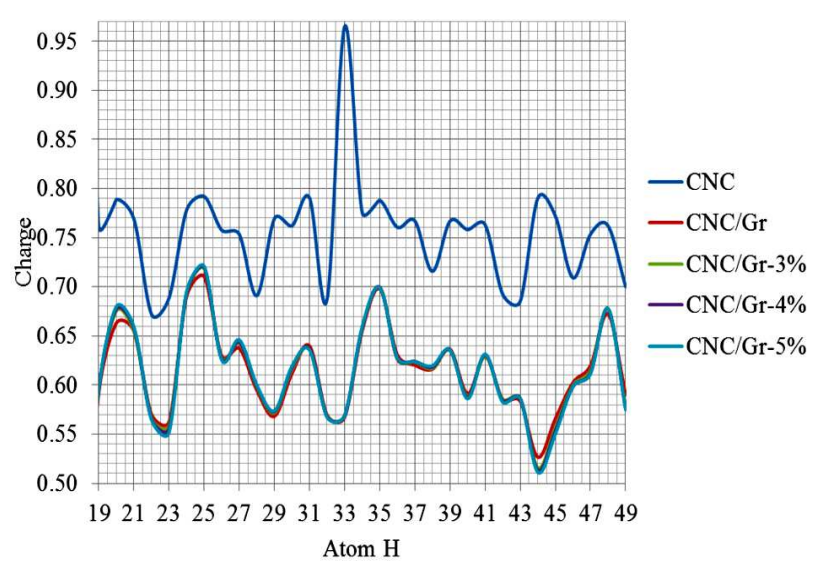

Fig. 6. Values of the charge in a vicinity of the hydrogen core for systems $1,4-7$ 
Table 4. Values of the charge in a vicinity of the oxygen core $(\mathrm{O})$ with a radius of $1.2 \AA$

\begin{tabular}{|c|c|c|c|c|c|}
\hline Atom & $\mathrm{CNC}$ & $2 \mathrm{CNC} / \mathrm{Gr}$ & $2 \mathrm{CNC} / \mathrm{Gr}-3 \%$ & $2 \mathrm{CNC} / \mathrm{Gr}-4 \%$ & $2 \mathrm{CNC} / \mathrm{Gr}-5 \%$ \\
\hline 50 & -2.823 & -8.064 & -6.683 & -6.622 & -6.579 \\
\hline 51 & -0.222 & -3.342 & -2.485 & -2.477 & -2.470 \\
\hline 52 & -0.797 & -4.345 & -3.343 & -3.327 & -3.302 \\
\hline 53 & -0.902 & -5.303 & -4.327 & -4.337 & -4.349 \\
\hline 54 & -0.488 & -3.234 & -2.422 & -2.413 & -2.402 \\
\hline 55 & -2.589 & -7.755 & -6.448 & -6.406 & -6.369 \\
\hline 56 & -2.666 & -8.129 & -6.849 & -6.842 & -6.832 \\
\hline 57 & -5.633 & -5.448 & -4.425 & -4.391 & -4.357 \\
\hline 58 & -0.807 & -5.345 & -4.336 & -4.323 & -4.311 \\
\hline 59 & -0.426 & -3.263 & -2.541 & -2.505 & -2.468 \\
\hline 60 & -2.885 & -8.777 & -7.644 & -7.727 & -7.821 \\
\hline 61 & -2.646 & -7.970 & -6.631 & -6.604 & -6.576 \\
\hline 62 & -0.640 & -4.216 & -3.287 & -3.290 & -3.293 \\
\hline 63 & -0.794 & -4.488 & -3.567 & -3.570 & -3.571 \\
\hline 64 & -0.760 & -4.645 & -3.899 & -3.949 & -3.975 \\
\hline 65 & -0.446 & -3.922 & -3.297 & -3.321 & -3.317 \\
\hline
\end{tabular}

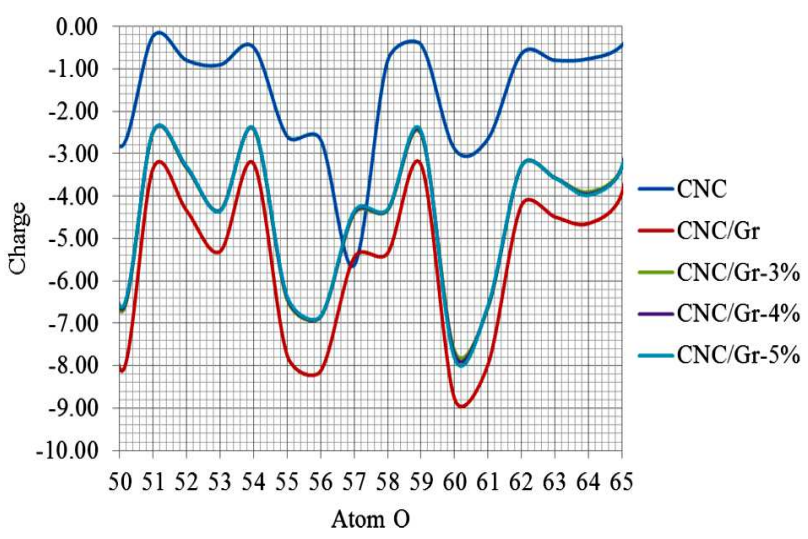

Fig. 7. Values of the charge in a vicinity of the oxygen core for systems $1,4-7$

nanocellulose/graphene composites and under their mechanical impact compared with the charges on all cores of an isolated nanocellulose fiber fragment. The exception is the behavior of an oxygen atom that is closest to graphene. The core charge on the oxygen atom begins to increase under a mechanical impact.

\section{Conclusions}

The spatial distribution of valence electrons, the density of electron states, the band gap, the valence band width, the values of charge in the vicinity of the atomic cores have been computed by meth- ods of the electron density functional theory and the first-principles pseudopotentials for the cellulosebased model composite structures under a mechanical impact.

The values of the charge in vicinities of the carbon cores, hydrogen and oxygen cores of nanocellulose decrease for all nanocellulose/graphene composites and their mechanical impact.

The maximum of the valence electron density of the cellulose-based composite is concentrated around cellulose for different composites under different mechanical impacts.

The electronic properties of the composite structures based on nanocellulose can be controlled, by changing the distance between the layers of composite components, which happens during the mechanical compression or stretching. This is a physical mechanism for using nanocellulose/graphene composites as electromechanical sensors.

1. S. Ummartyotin, M. Sain. Cellulose Composite for Electronic Devices (Thammasat Univ., 2016).

2. HORIZON 2020 - Work Programme 2016-2017. Crosscutting activities (Focus Areas), PILOTS-05-2017: Paperbased electronics.

3. L. Valentini, M. Cardinali, E. Fortunati. Nonvolatile memory behavior of nanocrystalline cellulose/graphene oxide composite films. J. M. Appl. Phys. Lett. 105, 153111 (2014).

ISSN 2071-0194. Ukr. J. Phys. 2018. Vol. 63, No. 9 
4. Chaoyi Yan et al. Highly stretchable piezoresistive graphene-nanocellulose nanopaper for strain sensors. Adv. Materials 26 (13), (2014).

5. Abdullahil Kafy et al. Cellulose/graphene nanocomposite as multifunctional electronic and solvent sensor material. Mat. Lett. 159, 20 (2015).

6. Ab initio calculation [E-resource] - Mode access to the resource: http://sites.google.com/a/kdpu.edu.ua/calculationphysics.

7. N. Yakovkin, P.A. Dowben. The problem of the band gap in lda calculations. Surface Rev. Lett. 14 (3), 481 (2007).

8. E.S. Kryachko, E.V. Ludeña. Density functional theory: Foundations reviewed. Phys. Rep. 544, 123 (2014).

9. Claudia D. Simao, Juan S. Reparaz, Markus R. Wagner, et $a l$. Optical and mechanical properties of nanofibrillated cellulose: Toward a robust platform for next-generation green technologies. Carbohydrate Polymers 126, 40 (2015).

Received 19.04.18
Р.М. Балабай, А.В. Здещии

НАНОЦЕЛЮЛОЗА

ЯК ОСНОВНИЙ КОМПОЗИТНИЙ КОМПОНЕНТ ЕЛЕКТРОМЕХАНІЧНИХ ДАТЧИКІВ

Р е з ю м е

Методами теорії функціонала електронної густини та псевдопотенціалу із перших принципів, використовуючи авторський програмний комплекс, було розраховано просторові розподіли густини валентних електронів, густину електронних станів, ширину забороненої зони, валентної зони та значення зарядів в околі ядра атомів для модельних композитних структур на основі наноцелюлози під механічним впливом. Встановлено, що електронні властивості структур на основі наноцелюлози можна регулювати, наприклад, шляхом змін відстаней між шарами композитних компонентів при механічному впливі. 